Revue d'histoire de l'Amérique française

REVUE D.HISTOIRE DE L'AMÉRIQUE FRANÇAISE

\title{
Consultation des vieillards et petite histoire
}

\section{Victor Tremblay}

Volume 3, numéro 2, septembre 1949

URI : https://id.erudit.org/iderudit/801548ar

DOI : https://doi.org/10.7202/801548ar

Aller au sommaire du numéro

Éditeur(s)

Institut d'histoire de l'Amérique française

ISSN

0035-2357 (imprimé)

1492-1383 (numérique)

Découvrir la revue

Citer cet article

Tremblay, V. (1949). Consultation des vieillards et petite histoire. Revue d'histoire de l'Amérique française, 3(2), 172-178.

https://doi.org/10.7202/801548ar d'utilisation que vous pouvez consulter en ligne.

https://apropos.erudit.org/fr/usagers/politique-dutilisation/ 


\section{CONSULTATION DES VIEILLARDS ET PETITE HISTOIRE ${ }^{1}$}

Le sujet qu'on m'invite à traiter ici est la "Méthode pour la cueillette orale de la petite histoire"; je l'intitulerais volontiers: "Méthode pour la consultation des "archives vivantes".

J'ai le droit de penser qu'en m'assignant ce sujet; $M$. le chanoine Groulx a voulu rendre un hommage amical et délicat à ce qu'a fait la Société Historique du Saguenay, en même temps qu'il a voulu faire servir à l'utilité de tous, les résultats d'une expérience qui n'est plus au stage d'essai.

Il s'agit de la consultation des anciens, de ceux qui ont vécu quand nous n'étions pas la, nos vieillards, ceux qu'un des mots heureux de l'abbé Albert Tessier a appelés "les archives vivantes".

Dès son origine notre société d'Histoire régionale a adopté ce procédé d'information, dont l'emploi avait même précédé sa fondation. C'est par la consultation des vieillards que j'ai commencé moimême, il y a vingt-neuf ans, le travail que continue la Société Historique du Saguenay.

La consultation des anciens a chez nous une importance particulière.

La Région du Saguenay, où la plupart des paroisses ont moins de cent ans, a l'avantage de posséder encore, dans presque toutes les localités, des survivants de la première heure ou du moins de la première génération, des témoins des débuts et de toutes les étapes de son développement. C'est une ressource précieuse, que nous ne pouvons pas négliger.

Et comme les relations écrites sont relativement très rares dans ces périodes de débuts où les colonisateurs ont trop à faire pour prendre

1. Travail présenté a la réunion générale de l'Institut d'Histoire de l'Amériquo frangaise (19 mars 1949). 
le temps d'écrire, nous aurions bien peu de sources d'information sur l'effort prodigieux qui nous a valu notre Saguenay, si nous n'avions pas les rapports de ces témoins qui l'ont vécu. C'est donc pour nous une ressource indispensable, que nous n'avons pas le droit de négliger.

Sans doute le récit d'un vieillard n'est pas le dernier mot de l'Histoire; aussi n'est-ce pas en ce sens que nous l'apprécions. Mais c'en est un incomparable premier mot: c'est lui qui nous initie aux faits, qui en démêle la trame et souvent en révèle les causes cachées; c'est lui qui surtout nous donne la vraie physionomie du passé. Et "Ia vraie physionomie du passe", c'est cela que doit refléter l'Histoire, si elle veut être l'expression du vrai.

Un phénomène que j'ai moi-même constaté est celui-ci: après la consultation d'un ancien on a la satisfaction d'avoir appris des choses intéressantes; quand on en a consulté cinq ou six c'est comme un monde qui se révèle, on découvre avec une surprenante netteté la physionomie des temps où ils ont vécu. C'est que le vieillard qui parle sans calculs de ce qu'il a vu et vécu, dépeint ainsi le passé tel qu'il fut, en traits vivants et réels; sans autre secours que les documents nous risquons de n'entrevoir ce passé que sous un jour incomplet et plus ou moins déformé par les clichés de notre temps. La Tradition orale captée à sa source est le correctif nécessaire, comme elle est souvent la clef de la documentation écrite.

La consultation des vieillards est tout un art. Travail facile en lui-même, il exige des précautions et des méthodes qui sont conditions du succès.

Il faut tenir compte de l'age et de ses exigences;

- il faut éviter d'influencer les récits ou les dires;

- il faut inspirer la confiance et prévenir ce qui peut fermer la bouche aux vénérables interviewés;

- il faut noter de manière à assurer l'exactitude et la clarté du compte rendu.

C'est évident qu'il ne faut pas faire subir à un octogénaire une sorte d'interrogatoire ressemblant à un "examen oral" de collège.

Dès l'abord, il faut lui donner l'impression d'une chose facile, toute simple, qui n'a rien d'une corvée ou d'une enquête judiciaire. 
- Les vieillards sont généralement réservés, souvent timides. Les aborder avec une solennité d'officiel - ou avec une allure trop camarade -, c'est les paralyser. Ainsi, se présenter avec une formule comme: "Vous allez me raconter votre vie", "Je veux vous faire conter tout ce que vous savez...", ou quelque chose de semblable, cela peut "couper la façon" au moins timide des vieillards.

La plupart n'osent pas parler par crainte d'être "gazettés"; cette pensée les terrifie. Et il arrive souvent qu'ensuite, quand ils ont dit beaucoup de choses et qu'ils ont la conviction d'avoir intéressé, ils sentiront un désir secret de voir cela imprimé; même ils le diront. Plus d'un nous a demandé: "Quand est-ce que va sortir le livre..?"

Il faut éviter aussi de procéder par questions directes et surtout de discuter. On peut bien, à propos, insister un peu, pour s'assurer que notre vénérable interlocuteur ou interlocutrice ne se trompe pas par distraction, mais ne jamais les bloquer par des contradictions; ceci intimide et gêne. Les vieillards aiment généralement mieux se taire que discuter, et ils deviennent réticents si on a l'air de douter de la valeur de leurs dires. Mieux vaut prendre leur rapport, tel quel. S’il y a des erreurs, elles seront corrigées plus tard, soit au cours de la conversation soit par les études et les informations prises ailleurs.

Le mieux est d'amorcer la conversation par un détail, un fait du "temps passé" sur lequel on est sûr que le vieillard a quelque chose à dire. C'est une entrée en matière toute naturelle et facile; on prend intérêt à ce que le vieillard a dit.., on sort le carnet pour "prendre une note là-dessus", ...et on continue de noter. Un sujet en suggère un autre, appelle une explication, occasionne des révélations... Cela peut continuer indéfiniment. Plus tout cela a le ton et l'allure d'une conversation naturelle, plus le vieillard parle volontiers et sans se tromper ni s'ennuyer.

On fait bien d'avoir à l'esprit, ou sur une petite feuille à portée des yeux, les points qu'on aura besoin de faire éclaircir; on guette alors l'occasion et on amène la question.

Nous avons remarqué que dans ce travail les femmes réussissent mieux que les hommes. - Question de psychologie? de charme? d'habileté à faire parler? d'aptitude à saisir le détail ?... En tout cas c'est un fait. - Nous en avons qui sont vraiment merveilleuses: celle, par exemple, qui a recueilli le récit du mariage de Thomas Lessard, publié dans le premier numéro des "Archives de folklore". 
Une bonne manière, c'est d'être deux amis pour cette consultation. L'un cause et l'autre écrit. Celui qui cause n'a qu'à mener la conversation; mais il fait bien de surveiller la marche du crayon, pour retarder au besoin par des petites observations le récit du vieillard et donner ainsi à celui qui écrit le temps de noter à son gré. - Car en certaines choses (soit en raison de l'importance du détail, soit à cause de la manière particulière de parler et qui mérite citation textuelle) il faut rapporter les paroles mot à mot, ou presque mot à mot.

Quoi demander? - N'importe quoi. Tout. Car tout est à savoir, même les choses que l'on sait ou que l'on croit savoir; on aura ainsi, en plus du nouveau, des détails qui corroboreront ou corrigeront beaucoup de notions connues avec plus ou moins de certitude.

Qu'on ne se limite pas, de grâce, aux faits rares, exceptionnels ou extraordinaires ! On est porté à cela par l'habitude de s'intéresser aux nouvelles plus ou moins sensationnelles; mais ce n'est pas de cela que la vie est faite, pas plus dans le passé que dans le présent; et si l'histoire doit tenir compte des faits accidentels ou extraordinaires, elle doit avant tout rapporter la vie réelle ordinaire, celle qui explique le présent et qui est la trame véritable du passé.

Nous avons préparé, nous, à titre de suggestions, un questionnaire où sont énumérés et groupés les sujets qui peuvent entrer dans la consultation des anciens. C'est une aide et un guide. - J'ai apporté ici ce questionnaire. On remarquera qu'il invite à recueillir des détails généalogiques; c'est nécessaire pour "situer" le vieillard et pour connaître les autres qui ont vécu dans son milieu, partagé son éducation, continué son œuvre, et aussi qui peuvent prolonger ou compléter les renseignements que nous avons obtenus de lui.

Celui qui s'inspire de ce questionnaire doit éviter toutefois d'astreindre son vénérable interlocuteur à répondre selon l'ordre des numéros. Il faut se souvenir qu'un vieillard, même quand il est "bien conservé", n'est pas plus à l'aise qu'un jeune dans un cadre serré. La meilleure tactique, pour se faire dire beaucoup de choses, est d'exploiter à mesure le sujet qui donne du rendement, quitte à revenir sur les autres sujets à un autre moment. Souvent en laissant le vieillard parler tout librement de ce qui lui vient à l'esprit on obtiendra une foule de renseignements qu'on n'aurait jamais pensé soi-même à luj demander ou qui auraient échappé à sa mémoire à un autre moment. 
Nous insistons pour que les notes prises dans ces consultations soient rédigées comme si le vieillard parlait lui-même, ou plutôt comme il a parlé: "J'ai vécu... Mon défunt père... Je vous dirai ben..." etc.

Ce système a d'abord l'avantage de simplifier l'effort quand il s'agit d'écrire en vitesse ce que dit le vieillard. Les notes et les expressions passent ainsi tout droit de l'oreille au crayon. Ensuite cela rend mieux la manière de parler du personnage: détail qui ne manque pas de saveur ni de valeur historique. Nous l'avons vérifié bien des fois. Nous y tenons surtout parce que c'est le seul procédé sûr pour éviter les équivoques et les confusions.

Il est en effet impossible, en notant a la course, de formuler toujours la distinction entre "il" désignant celui qui parle et "il" désignant celui dont il parle. Confusion alors quand on vient à relire; confusion plus grande pour les autres qui n'ont pas assisté à la conversation. Tandis que si on écrit les mots mêmes de celui qui parle: je, moi, etc., on prévient automatiquement ce danger.

Les notes prises doivent être transcrites aussitôt que possible après l'entrevue. Pendant la consultation, on abrège forcément (a moins de connaître la sténographie); il faut donc transcrire proprement, clairement ces notes, en conservant ou en rétablissant la conversation au complet, avec les propres expressions du personnage consulté. La langage de nos vieux est lui-même un fait historique que nous devons enregistrer.

Il est bon, généralement, que les entrevues ne soient pas trop prolongées; vaut mieux qu'elles soient plutôt répétées. Une première conversation ne fait qu'éveiller le passé. Ensuite tout un monde de souvenirs qui se précisent, se complètent et s'avivent à mesure que l'esprit s'y arrête et les repasse, peut alimenter plus d'une entrevue.

A ce propos, qu'on nous permette de citer une expérience faite a Saint-F6́licien, par un de nos correspondants. Il eut l'idée, après avoir consulté une demi-douzaine d'anciens de sa paroisse, de les réunir et de les faire rappeler et commenter ensemble certains faits de "leur temps". Ce fut un succès: pour les vieux une veillée incomparable, pour notre enquêteur une vraie "critique scientifique de la tradition orale".

Tout cet exposé de méthode, que je viens d'esquisser, se résume en définitive à quelque chose de bien simple: 1 - donner au vieillard 
toute la chance de parler à l'aise et beaucoup; 2-noter comme il parle, afin de conserver exactement le style et le sens de ce qu'il dit.

Qu'on me permette d'ajouter une observation: c'est que la consultation des vieillards est autre chose qu'une agréable fantaisie, un sport d'original. - Ce que disent ces témoins que la Providence nous a laissés est infiniment précieux. Au vrai, partout comme au pays du Saguenay, la documentation écrite est une bien pauvre ressource pour connaître un monde disparu et, sous certains aspects, bien différent du nôtre. - C'est la constatation de cette insuffisance qui a fait naitre ces sciences spéciales qui trouvent le secret de faire parler les choses mortes: l'archéologie et la paléontologie, dont les créateurs ont été finement définis par Louis Veuillot: "des savants messieurs qui prétendent lire des inscriptions effacées écrites en langues inconnues sur des monuments détruits". Pour nous en rendre compte nous-mêmes, il suffit de prendre comme comparaison l'activité de notre époque, celle à laquelle nous sommes mêlés, et d'observer la part qui s'en trouve inscrite dans les documents, et même dans les journaux, qu'on accuse volontiers d'en être le miroir. De qui et de quoi parlent les gazettes, en regard de ce qui se fait ?... La vie, qui anime tout, n'a pas d'échos, parce qu'elle ne fait pas de bruit; c'est le tapage, l'exceptionnel, l'accident, et souvent la propagande intéressée, qui vont à l'impression.

Avec ce résultat que dans aucun pays ou à peu près, l'histoire ne rend compte exactement du passé. Chez nous, par exemple: notre histoire écrite présente bien ceux qui ont découvert notre pays, ceux qui ont présidé à la fondation de ses grandes ou petites villes, ceux qui l'ont évangélisé, défendu, administré ou exploité; elle ne nous montre pas ceux qui l'ont fait. Il existe quantité de choses importantes dont l'histoire ne donne pas l'explication: telles, par exemple, la conquête du sol, l'œuvre lente et insaisissable qui a transformé, humanisé ce pays, les œuvres de dévouement que sont la famille, les institutions, l'éducation.., et que d'autres choses. Ainsi, si l'histoire de notre pays est partiellement faite, celle de notre peuple ne l'est pas du tout, ni celle de son œuvre édifiante (dans les deux sens); ce peuple qui, comme la vie tenace, fait, refait et répare sans cesse ce que ses "grands hommes" trouvent souvent moyen de compromettre ou de dissiper. 
Si les faits ordinaires d'une vie d'homme ou de femme, recueillis avec probité et respect comme des parcelles précieuses de la vérité historique, peuvent corriger partiellement cette lacune; s'ils peuvent aider à saisir le véritable sens des événements qui ont fait du bruit et dont il faut tenir compte, ils sont un appoint non seulement intéressant mais très important pour la documentation de l'histoire, et nous avons raison de chercher les meilleures méthodes pour les recueillir.

Victor Tremblay, ptre

president de la Societe historique du Saguenay 